
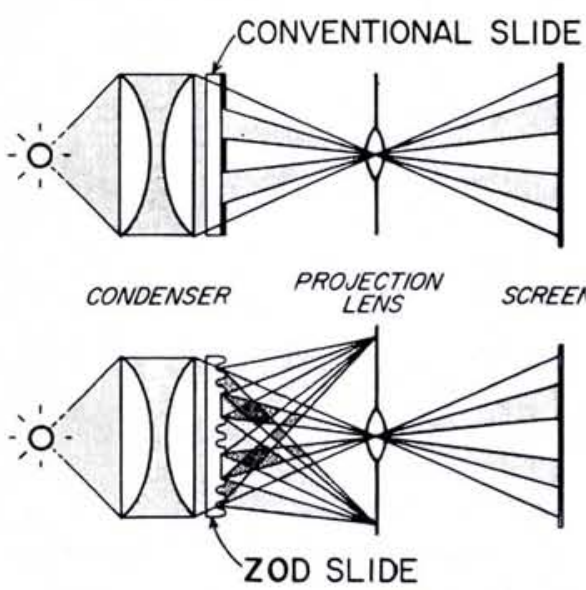

Fig. 3 - Read-out of conventional and $Z O D$ surface relief images in a standard projector.

aperture (see Fig. 3). Provided the grating period is sufficiently fine (typically about $1.5 \mu \mathrm{m}$ ) only the zero-order, undiffracted light passes through the lens to form an image on the screen these images have thus been named ZOD images (from Zero Order Diffraction).

Under white light illumination, the intensity and spectral composition of the light transmitted in the zero-order is determined by the profile and amplitude of the relief grating structure. Black and white images are reproduced using a crossed sinewave relief. For colour, squarewave gratings of different depths generate the yellow, magenta and cyan primaries for a subtractive colour scheme. Fig. 4 shows SEM enlargements of one of these three embossed plastic layers, which are superimposed to form such a tripack ZOD colour image. In fabrication, durable metal masters of the required relief structures are produced by photolithographic and electroplating techniques, and are then used for hot embossing into clear plastic sheet such as PVC.

The main application of ZOD image technology is in micropublishing, where information (journals, catalogues, price lists, etc.) is distributed in microfiche format. The system offers the valuable combination of full compatibility with existing display equipment, together with a low cost per copy for colour and black and white embossed replicas in volume. Resolution is similar to that of conventional micrographic recording materials and colour quality is comparable with that of colour microfilm emulsions. Since they are relief structures, ZOD images do not bleach or fade and are thus ideal for the archival storage of colour images - an application for which alternative recording media are currently not sufficiently stable. Diffractive structures such as these may also find applications as colour filters and beam splitters in areas where low cost and low weight are important considerations.

ZOD surface relief structures have also been used as the basis of a novel thermal recording scheme. The recording medium is plastic sheet pre-embossed with an optimized sinewave relief profile which would reconstruct black in read-out. A scanning, focused laser beam is used to write or contact-print images by selectively heating the plastic surface, resulting in plastic flow and a local levelling of the grating relief. Sensitization to the recording wavelength is achieved by incorporating dye into the plastic as a thin surface layer, enabling sensitization to any wavelength region in the visible to be readily achieved. Written areas are then coloured by the dye ; unwritten areas remain black. Applications include updateable microfiche and slides, and instant image recording.

A theoretical understanding of the diffraction properties of transmission phase gratings with grating constants and rectangular groove depths of the order of the light wavelength has been developed using rigorous diffraction theory. The scalar treatment based upon Huygen's and Kirchhoff approximations breaks down for such fine, deep gratings and the problem must be treated by numerical solution of Maxwell's equations. The theoretical predictions then agree closely with the measured diffraction efficiencies and can be used to optimize the rectangular groove grating parameters for a given filter application. Additionally, the properties and fabrication of multi-

\section{Scholarships at the University of Nijmegen}

The Faculty of Science of the University of Nijmegen is offering research fellowships to graduate students who hold a master's degree in science or its equivalent and who have at least one year's experience in research. Valid for 12 months they will initially be applicable to the academic year 1980-81.

Fellows are expected to carry out full-time research in one of the University's laboratories. A working knowledge of the Dutch language is not required but proficiency in English, French or German is necessary. The net monthly allowance will be h.FI. 1750.

There are openings in the: Dept. of Informatics and Mathematics Section; in the experimental Depts. of Atomic
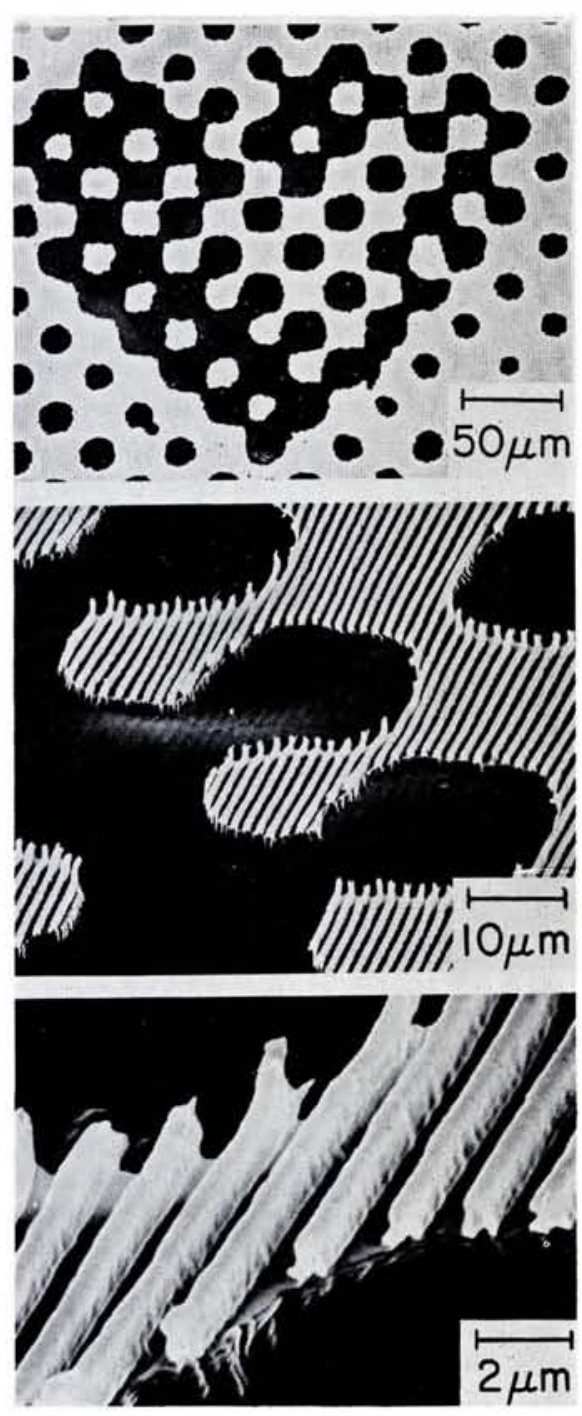

Fig. 4 - SEM enlargements of a colour ZOD image embossed in PVC.

level profiles are being investigated for applications in areas other than micro-imaging.

and Molecular Physics, High Energy Physics, Medical Physics and Biophysics, Solid State Physics and Solid State Chemistry; in the theoretical Depts. of Mechanics, High Energy Physics, Solid State Physics, and Astronomy; and in the Chemistry Section in the Depts. of Molecular Spectroscopy and Physical Chemistry.

Information concerning the research objectives and further details on the arrangements can be obtained direct from the Secretary of the Fellowship Committee, Faculty of Science, Toernooiveld, Nijmegen, The Netherlands. the EPS Secretariat in Geneva. Note that applications need to be made as soon as possible preferably even before 1 December, 1979. Application forms are available from 